\title{
In Defense of Newtonian Induction: Hume's Problem of Induction and the Universalization of Primary Qualities
}

\author{
Ori Belkind
}

November 1, 2018

\begin{abstract}
This paper aims to advance two claims. First, it aims to show that Hume's argument against the rationality of induction is sound. However, I claim that the conclusion does not follow merely from the self-defeating attempts to justify the rule of induction, unlike traditional readings of the argument. Rather, the skeptical conclusion must also take into account Hume's argument that the secret powers that are present in bodies and give rise to sensible qualities are unknowable. The paper's second aim is to show that Newtonian induction escapes Hume's secret powers argument, given that it includes a transductive inference, from observable phenomena to the powers present in the ultimate parts of matter. Consequently Hume's argument against the rationality of induction does not demonstrate the non-rational nature of Newtonian induction.
\end{abstract}

\section{Introduction}

This paper articulates a certain reading of Hume's argument against the rationality of induction. Unlike traditional interpretations of Hume's argument, mine takes his argument to involve two distinct sub-arguments; the first is that inductive inferences lack rational support. I term this sub-argument the No Rational Support Argument (NRSA) (Section 2). I also argue that Hume uses a second subargument, one I term the Secret Powers Argument, or SPA, to undermine our belief in the rationality of induction. According to the SPA (examined in Section 3), we do not have access to the secret powers of bodies. The implication is that we have no method for securing our belief that the same 
sensible qualities are accompanied by the same secret powers. Commentators normally focus on the NRSA without paying enough attention to the SPA. The consequence of this neglect is that most commentators assume that Hume's skepticism ought to follow from the NRSA alone, or, in other words, that the mere lack of rational justification for induction implies that induction is unjustified. I claim, however, that the NRSA is insufficient for deriving Hume's skeptical conclusion and that only by considering the SPA does the skeptical conclusion follow.

The further aim of my paper is to demonstrate that a Newtonian form of induction presupposes a transductive argument, according to which qualities of composite entities are shown to be reducible to qualities of their ultimate parts (Section 4). My claim is that Newton's transductive argument undermines Hume's SPA. This implies that Hume's skeptical conclusion lacks the bite required to directly undermine Newtonian induction (Section 5).

\section{The No Rational Support Argument}

There is no overall agreement among interpreters regarding the purpose of Hume's argument and the nature of his achievement. The widespread view among philosophers is that Hume has successfully articulated a powerful argument against the rationality of inductive inferences. Popper $(2003,6)$ is a prominent example of someone who thinks that Hume's argument is insurmountable; Popper concludes that one should adopt a hypothetico-deductive method of scientific inquiry. Some commentators claim that Hume's aim was not to articulate a skeptical conclusion regarding induction but merely to point out that induction does not fall under the cannon of "demonstrative" or deductive reasoning (Beauchamp and Mappes, 1975; Beauchamp and Rosenberg, 1981; Broughton, 1983; Arnold, 1983). In contrast, other commentators (e.g., Garrett (1997, 87-91), Millican (1998, 2012)) have argued that the non-skeptical reading seems incongruent with Hume's attempt to undermine the arguments that could potentially be given in support of inductive conclusions. I agree with the skeptical reading of Hume's argument, and my aim in this paper is to show that one ought to draw a skeptical conclusion regarding probable reasoning, as long as such probable reasoning can be modeled in the way Hume models it.

According to the standard reading, Hume aims to undermine the epistemic warrant that is provided by the inductive inference. But some commentators (Garrett, 1997) take Hume's argument to be directed at showing that no cognitive faculty of the human mind is capable of forcing the inductive 
conclusion. The latter descriptivist interpretation of Hume's argument finds Hume to be primarily concerned with developing a psychological theory of mind. It sees the skeptical argument as directed against the idea that such a theory can include some cognitive faculty other than the imagination that is able to force the inductive conclusion. My reading goes beyond the descriptivist account because I hold that the the lack of epistemic warrant follows only if one considers the SPA. But the SPA is not about the cognitive faculties of the knower; rather, it explores a metaphysical possibility that shows the potential unreliability of inductive procedures.

Hume begins his discussion with a distinction between Relations of Ideas and Matters of Fact (E 4.1, SBN 25). ${ }^{1}$ There are two criteria for differentiating between relations of ideas and matters of fact. The first criterion concerns the relation between the truth of the proposition and existent things. If the truth of the proposition is determined by the mere operation of the mind and is independent of what is anywhere existent in the universe, it is a relation of ideas. If its truth is dependent on existent things, it is a matter of fact. The second criterion Hume articulates for distinguishing between relations of ideas and matters of fact is concerned with the modality of the truth of the proposition. The contrary of a relation of ideas leads to a contradiction, while the contrary of a matter of fact does not; that is, one is able to conceive of the contrary and imagine its possibility.

Hume argues that all matters of fact that concern external objects that are not immediately present to our senses must rely on relations of cause and effect (E 4.4, SBN 26-7). This implies for Hume that any matter of fact that pertains to objects that are not immediately present to the senses presupposes knowledge of causal relations.

Hume introduces skeptical doubts about propositions expressing matters of fact, by introducing doubts into causal relations. The argument is bifurcated in that the proposition expressing a causal relation may be either a relation of ideas or a matter of fact. For each type of proposition, one would expect different types of reasoning given in support of it. A relation of ideas is given a priori justification, while a matter of fact is provided justification based on experience. Hume claims that neither type of justification is suitable to ground a causal claim.

\subsection{Why a priori reasoning cannot justify causal claims}

Hume asserts that a causal claim is not a relation of ideas (E 4.6, SBN 27). There are various ways in which Hume attempts to demonstrate this claim, but they all boil down to the notion that the cause

\footnotetext{
${ }^{1}$ E refers to Hume (1975) and SBN refers to Hume (1978). Quotes from taken from https://davidhume.org/.
} 
is separable from the effect. If it is conceivable in the imagination that the cause exists without the effect that follows, the relation between cause and effect cannot be a relation of ideas.

It is important for Hume to stress that even laws of nature-for example, the conservation of quantity of motion-cannot be treated as relations of ideas:

But to convince us that all the laws of nature, and all the operations of bodies without exception, are known only by experience, the following reflections may, perhaps, suffice. ... The mind can never possibly find the effect in the supposed cause, by the most accurate scrutiny and examination. For the effect is totally different from the cause, and consequently can never be discovered in it. Motion in the second billiard ball is a quite distinct event from motion in the other. A stone or piece of metal raised into the air, and left without any support, immediately falls; but to consider the matter a priori, is there anything we discover in this situation which can beg the idea of a downward, rather than an upward, or any other motion, in the stone or metal? (E 4.9, SBN 29)

We see that, while the initial target of Hume's remarks were causal relations, the discussion quickly turns to fundamental laws of nature and to Newtonian science. Hume argues that both the mechanical laws stemming from the conservation of quantity of motion (momentum) and the law of gravitation, cannot be justified using a priori reasoning; they are not dependent on the mere operation of thought, but must rely on experience.

Another way in which Hume shows that causal relations are not relations of ideas is by arguing that the effect cannot be derived from the cause using any a priori reasoning.

I shall venture to affirm, as a general proposition, which admits of no exception, that the knowledge of this relation is not, in any instance, attained by reasoning á priori; but arises entirely from experience, when we find, that any particular objects are constantly conjoined with each other. (E 4.6, SBN 27)

This suggests that the initial failure is in being able to demonstrate that a causal relation is a relation of ideas. If such a demonstration were possible, one would be able to derive the effect from the cause using some carefully constructed demonstration that is grounded in the ideas themselves. Perhaps this could be done by observing the cause and then, via some abstract inference, deducing the presence of the effect. 
Hume argues that it is not possible to find an a priori reasoning for a causal relation. According to Hume's account of rational demonstration, one is either directly aware of two ideas being inseparable in some way, or it is possible to construct a chain of reasoning, using distinct steps involving inseparable ideas, leading from one idea to another. ${ }^{2}$ In the Treatise, Hume argues that certainty in demonstration arises from particular relations that make the ideas inseparable:

All certainty arises from the comparison of ideas, and from the discovery of such relations as are unalterable, so long as the ideas continue the same. These relations are resemblance, proportions in quantity and number, degrees of any quality, and contrariety. (T 1.3.3.2, SBN 79$)^{3}$

These philosophical relations provide the underpinning of rational demonstrations. They must be present whenever a claim is shown to be "intuitively or demonstratively certain."

But the relation between cause and effect cannot be demonstrated in such a way. The ultimate proof that such a demonstration is not possible is that the idea of the cause is distinct and separable from the idea of the effect. Given that the two ideas in a causal relation are taken to be contiguous, they are present at distinct moments in time. And so, by definition, one can conceive of the cause without the effect materializing, and the two ideas are separable. Thus, it would not be possible to find any demonstration leading from the cause to the effect because that such a demonstration is based on linking pairs of inseparable ideas.

Section 4 will consider Newton's argument in support of laws of nature. At this point I would merely point out that Newton agrees that laws of nature are not discovered using intuitive or demonstrative reasoning, but instead ought to be derived from the phenomena with the help of some inductive procedure.

Hume claims that however successful a science might be in simplifying phenomena and reducing them to fundamental laws, knowledge of the ultimate grounds for these laws remains hidden from our understanding:

It is confessed, that the utmost effort of human reason is to reduce the principles, productive

of natural phenomena, to a greater simplicity, and to resolve the many particular effects into

\footnotetext{
${ }^{2}$ For Hume the inseparability of ideas is the foundation for demonstrative reasoning: "In order therefore to know, whether abstraction implies a separation, we need only consider it in this view, and examine, whether all the circumstances, which we abstract from in our general ideas, be such as are distinguishable and different from those, which we retain as essential parts of them" (T 1.1.7.3, SBN 18-9). See also De Pierris (2015, Section 2.2).

${ }^{3} \mathrm{~T}$ refers to the original edition of the Treatise.
} 
a few general causes, by means of reasoning from analogy, experience, and observation. But as to the causes of these general causes, we should in vain attempt their discovery; nor shall we ever be able to satisfy outselves, by any particular explication of them. These ultimate causes and principles are totally shut up from human curiosity. Elasticity, gravity, cohesion of parts, communication of motion by impulse; these are probably the ultimate causes and principles we shall ever discover in nature; and we may esteem ourselves sufficiently happy, if, by accurate enquiry and reasoning, we can trace up the particular phenomena to, or near to, these general principles. (E 4.12, SBN 30-1)

Thus Hume allows that science has a role in articulating general principles, such as the laws of motion and the law of gravity, to explain various phenomena. But he takes the "causes of the general causes"-that is, the causes that bring about these principles- as inaccessible to the human mind. The implication is that reason, by itself, is not able to discover the causes that ground laws of nature. ${ }^{4}$

\subsection{Why experience cannot ground causal claims}

Given that the causal claim is not a relation of ideas, it must ultimately be derived from experience.

But how would this derivation work? Hume describes the inference as follows:

...there is a certain step taken; a process of thought, and an inference, which wants to

be explained. These two propositions are far from being the same, I have found that such an object has always been attended with such an effect, and I foresee, that other objects, which are, in appearance, similar, will be attended with similar effects. (E 4.16, SBN 32)

So Hume is concerned with the inference one draws from "All observed As are Bs" to "All As are Bs". We shall refer to this inference as the Rule of Induction $(\mathbf{R I})$. The problem is that the reasoning seems to lack justification. This type of inference cannot be reduced to intuitive or demonstrative reasoning because of the initial part of the argument, according to which a causal relation is not a relation of ideas.

If one one seeks to justify inductive inferences using probable reasoning, one runs into circular justification: "To endeavour, therefore, the proof of this last supposition by probable arguments, or arguments regarding existence, must be evidently going in a circle, and taking that for granted, which is the very point in question" (E 4.19, SBN 35-6). How exactly is the circularity in justification created?

\footnotetext{
${ }^{4}$ See Schliesser $(2008,2009)$ for an evaluation of Hume's philosophy vis-a-vis Newton's work.
} 
Hume argues that in any inductive generalization, the implicit assumption is that the unexamined cases will be like examined cases, or that the future will resemble the past (E 4.19, SBN 35-6). We call this assumption the Principle of the Uniformity of Nature (PUN).

To describe the argument somewhat formally, the circularity of the argument seems to be concerned with the inductive inference, and the presuppositions that one might provide to justify the inference. The claim is that if we are to try to show why the RI is reliable it compels us to think of the PUN as the principle that might justify the reliability of RI. However, the PUN cannot be shown to be true using demonstrative reasoning. It is conceivable that unexamined cases will not be like examined cases. Thus, the PUN has to be justified using experience. It follows that the PUN can only be justified with RI. The justification is therefore circular and empty. ${ }^{5}$

The consequence of Hume's argument is that no rational support can be given in favor of inductive inferences. One can safely conclude that induction is not a rational inference. Hume provides a psychological theory of induction, according to which induction is based on certain associations of ideas carried out by the mind. Once we experience a correlation several times, a mental habit forms, and we expect the effect to be present every time the cause is experienced. The association between cause and effect is then explained by psychological processes and principles attributed to human nature. However, this sort of account is not a justification of the inductive inference, but an explanation of the natural conditions that give rise to it.

Hume seems to accept what De Pierris $(2015,5)$ calls a presentational-phenomenological model of logical demonstration. The idea is that there are relations between ideas that can be grasped intuitively. If a pair of ideas is inseparable, or it is not possible to conceive of the presence of one idea without the presence of the other, then the proposition describing the relation is a relation of ideas. Its truth is recognized a priori and it is not possible for it to be false. Furthermore, if a relation between two ideas is not grasped intuitively, it is still possible that one would be able to construct a demonstration - that is, a linked chain of pairs of ideas - in which the relation between each pair is grasped intuitively as inseparable. The various steps within a geometric proof might not be grasped intuitively together, but it may be broken down into distinct steps, each of which is intuitively grasped and leads from one idea to the next. In principle, one should be able to distinguish between knowledge,

\footnotetext{
${ }^{5}$ Commentators sometimes object to Hume's argument by claiming that turning to the PUN to support the RI amounts to the demand that the RI be turned into a deductive argument. That is, they claim that Hume commits himself to a deductivist conception of justification. However, it seems to me that this charge is misguided. One might say that introducing the PUN merely amounts to claiming that inductive inferences are reliable-that is, that they are likely to produce true results. See Millican $(1995,108)$ for a similar rebuttal.
} 
which is either intuitively or demonstratively certain, and probable claims, which lack the certainty of knowledge-claims.

In the Treatise, Hume suggests that, while one should in principle be able to distinguish between demonstrative and probable inferences (T1.4.1.1, SBN 180), there are doubts as to whether demonstrative reasoning actually achieves the certainty one normally attributes to it. If one were to actually construct a chain of reasoning, one would need to rely on human cognitive faculties to be able to link the chain of intuitive steps correctly. But there are reasons to doubt that humans have an infallible cognitive faculty. First, we need to rely on memory, and the assuredness that a pair of ideas that was perceived in the past as inseparable is in fact correctly remembered as inseparable. And the transition of awareness from one step of a demonstrative proof to another also requires never transitioning to ideas that were not linked by inseparable ideas. Because human cognitive faculties rely on some causal mechanism, the ability to proceed correctly with demonstrative reasoning can legitimately be seen as potentially unreliable ( $\mathrm{T}$ 1.4.1.2, SBN 183). Thus Hume thinks that demonstrative reasoning should be understood as dissolving into probable reasoning, since it can never achieve as a matter of fact the certainty that mathematicians or logicians normally attribute to it. ${ }^{6}$

Nevertheless, despite the skepticism Hume expresses about demonstrative reasoning, it is a skepticism that assumes a certain standard of demonstrative validity. Namely, Hume does accept that awareness of inseparable ideas guarantees the truth of a proposition expressing the relation. It is just that the human mind is limited, and can have in its direct awareness only a single pair of ideas at a time. Hume accepts a certain conceivability criterion: If it is possible to conceive of a pair of ideas as separable, the ideas are, in fact, separable. And if it is not possible to conceive of a pair of ideas as separable, they are, in fact, inseparable. Thus, the conceivability criterion suggests that for Hume, there is a standard for demonstrative reasoning, not achievable as a matter of fact, but one that takes the notion of demonstrative reasoning as a regulative idea.

While Hume is skeptical of demonstrative reasoning, this is a rather benign skepticism in comparison with his skepticism regarding the RI. Hume seems to accept the regulative ideal of demonstrative reasoning without attempting to provide further, second-order justification for it. He makes no attempt to justify the conceivability criterion or the notion that awareness of separability or inseparability guarantees that the ideas are, in fact, separable or inseparable. However, he does attempt to show that there is no such thing as a rational, inductive inference, even as a regulative notion. We

\footnotetext{
${ }^{6}$ See also De Pierris $(2015,2.4)$.
} 
seem to come up empty whenever we try to imagine how the RI might be taken to be rational; that is, we are not capable of reconstructing how the inference is informed by human reason, assuming there is a cognitive faculty that guides such inferences. The best we can do is uncover the psychological mechanisms that establish, by custom and habit, the associations between cause and effect. Yet what is unclear is why we should accept demonstrative forms of reasoning without second-order justification while requiring such justification of inductive forms of reasoning.

Ancient forms of skepticism argue that epistemic justification comes in three forms. In one possibility, justification leads to an infinite chain of reasons; if we take $q$ to justify $p$, it may be that this would only be possible if $q$ is justified by another proposition $r$, and so forth ad infinitum. Another possibility is that the chain of justification runs in a circle. The third possibility is that the chain of justification ends at a proposition that is not further justified. Either we accept this proposition dogmatically-that is, claim that some propositions are accepted without justification at all-or we take these propositions to be self-justified-that is, they are propositions that somehow are able to provide their own justification. This latter possibility is epistemic foundationalism. Clearly, in the case of rules of inference (or intuitive awareness of inseparability), neither infinite regress nor circular justification will do.

Since inductive inferences cannot be reduced to demonstrative reasoning, should we take them to require some additional justification? A comparison between probable and demonstrative types of reasoning ought to provide a proper context for evaluating the demand for justification. In the context of modern theories of logic, deductive reasoning is based on formal rules, which one can use to establish the validity of arguments. Consider, for example, modus ponens (MP), a rule of deductive inference. Consideration of MP immediately shows its validity. We normally do not require any further justification in considering the validity of this rule. In fact, we recognize that the rule supplies justification for deductive transitions, though we are not usually asked to supply arguments to justify the rule.

Carroll (1895) famously argued that the attempt to justify MP leads to an infinite regress of higher levels of MP rules, each intended to justify the MP rule of the lower level. If one were to demand additional justification for MP, such a demand would lead to infinite regress and no justification at all. But perhaps rules of inference like MP are rules that cannot be justified, or they are self-justified, or there is some entitlement one can be given in using them without providing additional justification. If one of these possibilities is the case, it follows that perhaps Hume should be skeptical of both RI 
and MP or of neither. But the preference for demonstrative reasoning should at least be explained. ${ }^{7}$

Hanna (2006, Ch. 3) argues that philosophical attempts to justify logical inferences (whether inductive or deductive) suffer from what he calls the "logocentric predicament." 8 This predicament arises from having to rely on the canons of logical inference in any attempt to justify the validity of logical inferences. The predicament seems to crop up in various philosophical accounts that justify logical inferences. For example, Quine famously objects to Carnap's conventionalist account of logical truths. The gist of Quine's critique is that in trying to apply a logical convention to particular instantiations of the convention, one needs to already take for granted the validity of the logical inference (Hanna, 2006, Sec 3.2). Hannah reviews various strategies for dealing with the logocentric predicament, that is, various accounts of how one can be entitled to use logical inferences without providing them second-order justification. These strategies normally dissolve the supposed difference Hume points out between inductive and deductive inferences, because if one is entitled to use deductive inferences without second-order justification, there is little reason not to grant such entitlement to inductive inferences. Hanna identifies six strategies for dealing with the predicament: logical prudentialism, logical communitarianism, semantic and epistemic holism, logical nonfactualism, logical instrumentalism, and logical cognitivism. In general, these strategies dissolve the difference between deductive and inductive inference because they appeal to non-logical facts in their explanation of the entitlement speakers have in using certain inferential tickets. For example, according to logical communitarianism, logical rules are the result of communities implicitly adopting certain forms of communication. But this implies that we can appeal to the norms of argumentation adopted by the community as the ultimate grounds for utilizing logical rules, opening the door to accepting both deductive and inductive rules on similar grounds (see for example Strawson (1952, 256-257) and Wittgenstein (1958, 134-136)). Or, in the case of semantic and epistemic holism, the validity of logical inferences is accepted as part of a web of beliefs, and its justification results from the coherence it forms in a whole range of propositions accepted by the community of inquirers, some logical and some factual. Such holistic accounts have provided a context where the difference between inductive and deductive reasoning seems to dissolve, because the inductive scheme of reasoning can be viewed as part of a coherent set of beliefs (this is explicitly argued for by Goodman (1983, 62-66)). According to the non-factualist approach, we can assume we are entitled to certain inferential schemes without being able to justify these schemes using

\footnotetext{
${ }^{7}$ See Perez Otero (2008) for a similar use of Carroll's paradox as an analogy for inductive justification.

${ }^{8} \mathrm{I}$ thank one of the reviewers for referring me to Hanna's succinct treatment of the logocentric predicament.
} 
empirical or a priori evidence. As long as we carefully extend those entitlements so as not to justify our prejudices, when no evidence exists to the contrary, and if the proposition has a cornerstone role in enabling a certain practice of inquiry, our acceptance of such propositions can be considered legitimate (see, e.g., Wright and Davies (2004)). We could then explain our entitlement to such propositions as expressing our trust in them. This provides opportunity to accept inductive rules of inference based on such attitudes of trust. The strategies of logical instrumentalism and logical cognitivism have also been used to dissolve the difference between inductive and deductive forms of reasoning. ${ }^{9}$

These attempts to take the RI as not requiring second-order justification ignore Hume's SPA, or the Secret Powers Argument. The reason the SPA is easy to ignore is that it is not a formal argument regarding the structure of RI, but an argument relying on the notion of power and the process by which sensible qualities come into existence. I claim in the following section that the argument is intended to provide a reason for thinking that $\mathbf{R I}$ is not reliable. Thus, evidence against the reliability of induction produces more skepticism than the claim that any justification of induction is circular.

\section{The Secret Powers Argument}

There is widespread agreement that Hume has shown that inductive inferences do not have rational justification. But a concern lingers over Hume's argument, which is: Why is justification required? Hume did not seek second-order justification for demonstrative reasoning. He took propositions expressing the relation between inseparable ideas to be intuitively or demonstratively certain. He explicated this with the help of the separability principle, but he did not attempt to justify the principle; and yet he did require second-order justification for inductive arguments. Probable arguments require a distinct notion of strength, of a weaker form than deductive validity, because the true premises in an inductive argument do not guarantee the truth of the conclusion; they merely make it probable or likely to be true. Yet, perhaps it is still possible to think of $\mathbf{R I}$ as a rule of reasoning accepted without justification in parallel with deductive rules of inference, which are accepted without second-order justification.

I take it that the disparity between inductive and deductive reasoning is bolstered by another argument, which I shall refer to as the Secret Powers Argument (SPA). Assume we have two proposi-

\footnotetext{
${ }^{9}$ Lange (2008, Section 5) also mentions externalist means or taking a naturalist approach towards epistemology to justify inductive schemes of inference (e.g., Van Cleve (1984) and Perez Otero (2008)). If we presume that there are external reasons or causes that show that $\mathbf{R I}$ is reliable, the rule circularity involved in the justification of induction is not a vicious epistemic circularity.
} 
tions, $p$ and $q$, and both are candidates for propositions we would like to accept without justification. Any attempt to justify the two propositions leads to self-defeating forms of justification. It seems as if we are faced with the choice of accepting both propositions or neither, depending on our epistemic attitude. The situation will cease being the same with regards to both propositions if we have reasons for thinking that one of the propositions might be false. If, for example, we find evidence that there are circumstances in which $p$ is likely to be false, but we cannot conceive of analogous circumstances where $q$ is false, then the self-defeating justification would be telling against $p$ while it would not be telling against $q$.

In the Treatise, Hume introduces a further argument against the PUN, or the claim that unexamined cases must resemble examined cases.

It may, perhaps, be said, that after experience of the constant conjunction of certain objects, we reason in the following manner. Such an object is always found to produce another. 'Tis impossible it cou'd have this effect, if it was not endow'd with a power of production. The power necessarily implies the effect; and therefore there is a just foundation for drawing a conclusion from the existence of one object to that of its usual attendent. This past production implies a power: The power implies a new production: And the new production is what we infer from the power and the past production. (T 1.3.6.8, SBN 90)

Hume holds that the correlation between two sensible qualities $A$ and $B$ is unexplained, or a mysterious coincidence, unless we could attribute the body in which $A$ inheres a power to produce quality $B$. The power attributed to a body is a certain capacity to produce effects. The attribution of a power to the body can be considered the metaphysical equivalent of taking the PUN to be valid. If the examined case in which $A$ was present produced $B$, then we may conclude that bodies with sensible qualities $A$ have the power to produce $B$. Then, the next time we observe a body with $A$, from the fact that we can attribute to it the power to produce $B$, we can predict that $B$ will be present as well. However, Hume points out that the power itself is unobservable - what is present to the mind is the impression associated with $A$, and the impression does not reveal the power that generated the impression; it is merely the effect of that power.

Your appeal to past experience decides nothing in the present case and at the utmost can only prove, that that very object, which produc'd any other, was at that very instant endow'd with such a power; it can never prove, that the same power must continue in 
the same object or collection of sensible qualities; much less, that a like power is always conjoin'd with like sensible qualities. Shou'd it be said, that we have experience, that the same power continues united with the same object, and that like objects are endow'd with like powers, I wou'd renew my question, why from this experience we form any conclusion beyond those past instances of which we have had experience. (T 1.3.6.10, SBN 91)

Hume's claim is that there is no reason to suppose that the same powers that correlate with observable quality $A$ would continue to be present in other bodies of the same quality $A$. This supposition amounts to the expectation that like sensible bodies are always conjoined with like powers. But nothing prevents us from thinking that like sensible qualities are conjoined with distinct powers, and so, nothing underwrites our expectation that future objects with sensible quality $A$ will carry the same power that produced sensible quality $B$. It should be clear that the power Hume is considering is not the causal power of quality $A$ to produce quality $B$. We may distinguish between the power to produce $B$ and the sensible quality $A$ because we do not know the internal constitution of bodies within which $A$ was observed nor do we know what exactly it is that produced $B$. The notion of power is the idea that something is present in a body that can produce sensible quality $B$.

In the Enquiry, Hume articulates a similar argument, which thinks of powers as involving the 'secrets' of nature. Thus, in the Enquiry, Hume stresses the difference between qualities "on the surface," that is, qualities belonging to macroscopic bodies that are observable to the senses, and the structure of those bodies and powers that exist in the hidden parts:

It must certainly be allowed, that nature has kept us at a great distance from all her secrets, and has afforded us only the knowledge of a few superficial qualities of objects; while she conceals from us those powers and principles on which the influence of those objects entirely depends. Our senses inform us of the colour, weight, and consistency of bread; but neither sense nor reason can ever inform us of those qualities which fit it for the nourishment and support of human body. Sight or feeling conveys an idea of the actual motion of bodies; but as to the wonderful force or power which would carry on a moving body for ever in a continued change of place, and which bodies never lose but by communicating it to others of this cannot form the most distant conception. But notwithstanding this ignorance of natural powers and principles, we always presume, when we see like sensible qualities, that they have like secret powers, and expect that effects, similar to those which we have experienced, will follow from them... It is allowed on all hands that there is no 
known connexion between the sensible qualities and the secret powers; and consequently, that the mind is not led to form such a conclusion concerning their constant and regular conjunction, by anything which it knows of their nature. (E 4.16, SBN 32-4)

The argument seems to be that the simplest sensible quality is produced by unknown powers. This is a principled form of ignorance - we know the effects of powers in bodies because we can perceive the impressions that are produced by them, but we do not know the powers that produced the impressions. We know the colour, the weight, and consistency of bread, but the power to nourish us remains hidden from the senses. We know the effects of the force of inertia, but we do not know the force of inertia itself because we cannot penetrate into the nature of the body beyond the effects that were produced and observed.

The secret nature of powers opens the door to conceiving of failures of the principle "like sensible qualities have like secret powers". That is, we may conceive of ways in which this connection between effects and the powers that produce them is false. We can conceive of two distinct secret powers producing similar sensible qualities, a possibility we cannot rule out. It may be that two distinct powers present in two loaves of bread produced all the same sensible qualities - the same colour, the same weight, and the same consistency of bread. Yet, it may be that in one loaf of bread those secret powers would produce nourishment and in another loaf of bread some other secret powers would not produce nourishment. In that case, it is always possible that the same impressions (or the effects of certain powers) were produced by distinct powers.

Given the possibility of divergent secret powers that produce the same sensible quality, we can distinctly and clearly conceive of those cases where induction is likely to fail. These possibilities of failure do not merely suggest that we can conceive of $A$ as being present without $B$. The possibility of $A$ being present without $B$ is merely a conceptual possibility; all it tells us is that the causal relation between $A$ and $B$ cannot be derived using intuitive or demonstrative reasoning. Nevertheless, the possibility of distinct powers producing similar sensible effects provides an account of how inductive reasoning could lead to a false conclusion. Some secret power may have $A$ appear and then also generate $B$, while another may have $A$ appear without $B$. That is, it is possible to conceive of a definite failure of the inductive procedure despite nature being entirely uniform and consistent because there is always an unbridgeable distance between observable impressions and the secret powers in bodies that produced them. ${ }^{10}$

\footnotetext{
${ }^{10}$ Schliesser (2017) in a blog post discusses the role of the SPA in Hume's argument against induction. Schliesser places
} 
The SPA provides Hume with independent reasons for thinking that inductive inferences are not reliable:

As to past experience, it can be allowed to give direct and certain information of those precise objects only, and that precise period of time, which fell under its cognizance: but why this experience should be extended to future times, and to other objects, which for aught we know, may be only in appearance similar; this is the main question on which I would insist. The bread, which I formerly eat, nourished me; that is, a body of such sensible qualities was, at that time, endued with such secret powers: but does it follow, that other bread must also nourish me at another time, and that like sensible qualities must always be attended with like secret powers? (E 4.16, SBN 32-4)

The SPA provides us with distinct reasons for thinking that inductive generalizations could lead to false conclusions, even if we hold nature to be uniform - or, in other words, even if we imagine the same powers always yield the same effects. But if the SPA provides reasons for thinking that the RI is unreliable, it goes further than the NRSA, which merely indicates to us that there are no means of justifying the RI since justification will end up being circular. The SPA therefore separates the RI from any deductive reasoning, in that it describes the exact circumstances in which the argument fails.

In this sense, we see that the SPA works in tandem with the NRSA, and that neither argument is sufficient by itself for securing Hume's conclusion. The SPA merely shows when inductive generalizations could fail, but it would not provide ultimate grounds for taking the $\mathbf{R I}$ to be unjustified if other justification might be given in its support. On the other hand, the NRSA, while valid, cannot ground the non-rational nature of the RI, given that it is possible to treat basic rules of inference as the bedrock of rational justification, so they themselves do not require independent justification. It is both the SPA and the NRSA that lead to Hume's skeptical conclusion, but neither is sufficient.

The problem with the SPA is that, to make it plausible, one has to entertain some potential theories of matter and the metaphysical notion of power or action so as to conceive of various possibilities of secret powers. The argument lacks the clean non-specific structure possessed by formal analyses of rules. Thus, the articulation of a potential failure of the RI cannot completely abstract from the metaphysical or scientific context within which the argument is made. It is therefore tempting to

Hume's argument in relation to the explanatory models of Early Modern philosophy, where essences, inherent natures, or active principles are taken to be the origin of the effects of natural bodies on other bodies. Thus we may think of Hume's argument as undermining the explanatory ideals of 17 th- and 18th-century science. 
lay the SPA aside, given that it is not an argument concerning the formal structure of RI. In the vast majority of reconstructions of Hume's argument, the SPA is not mentioned as an independent component in Hume's reasoning, and it is ordinarily taken to give further support for undermining the PUN, even though the NRSA by itself is not sufficient to drive Hume's conclusion.

\section{Newtonian Induction}

We have seen that Hume's argument is directed at Newtonian science, and his claims purport to show the non-rational nature of inductive inferences. It is likely that Hume speaks to the newly established image of science. The view, promoted by the Royal Society, was that a true science is based on the Baconian method. Science must begin with a direct examination of the phenomena, and use a methodical and slow process of deducing scientific propositions from the phenomena. Newton articulates his commitment to induction in Newton's Rule IV for the Study of Natural Philosophy:

In experimental philosophy, propositions gathered from phenomena by induction should be considered either exactly or very nearly true notwithstanding any contrary hypotheses, until yet other phenomena make such propositions either more exact or liable to exception.

(Newton, 1999, 796)

Hume does not think that there is any alternative to the inductive method. He himself can be taken to articulate a Newtonian-like project for the moral sciences, where rules of association for ideas are naturalized and taken to be analogous to the gravitational force that generates attraction between bodies.

What does Newton mean by induction, and can we take Hume to be attacking the same method utilized by Newton? By undermining the causal relations that underwrite matters of fact, Hume is introducing a skeptical argument that supposedly undermines all ampliative reasoning that might be used by scientific theories. The target is any necessary connection that scientific propositions might form between sensible qualities, ones that are not derived from intuitive or demonstrative reasoning. Hume's argument models such ampliative reasoning as follows: First we observe a regularity in nature; namely, we observe two sensible qualities conjoined together in repeated observations and experiments. Then we conclude that the two sensible qualities are linked by a necessary connection, and that it is not possible for any future occurence of the first sensible quality to be present without the second. 
For Hume, it is this simple template for inductive reasoning that underwrites all scientific reasoning. ${ }^{11}$

However, Newton's various arguments in his published work are quite complex. He often presents his arguments in deductive, mathematical style, constructing proofs and relying on axioms and derived propositions to articulate his theories. Newton's reasoning often seems more abstract than the simple enumerative induction depicted by Hume. His work in the Opticks most closely resembles Hume's account of the scientific inference. Newton describes various experiments he performed, wherein he finds a necessary connection between a ray of light's angle of refraction in moving from one medium to another and the ray's color. While the angle of refraction is not a sensible quality, it is not difficult to deduce this angle from observations and from basic knowledge of the motion of rays of light. Once the basic connection between the angle of refraction and color is articulated, Newton deduces many other propositions from this basic proposition. Newton thinks that his conclusions are more certain than mere Baconian generalizations because the reasoning from experiments is mediated via geometric theorems and propositions and is based only on observations and not hypotheses or conjectures. Thus, given that the angle of refraction is defined exactly by Euclidean geometry and given that no hypotheses regarding the nature of light are employed, one can draw more precisely and more exactly various conclusions from the basic axiom of the Opticks. This does not imply that Newton believes his conclusions carry the same certainty as mathematical certainty since physical principles are being relied upon in the derivations. He does think that the connection between the angle of refrangibility and color is demonstrated with certainty, that is, without relying on conjecture or hypothesis (see Walsh $(2017,873-876))$. Nevertheless, there is no conflation between the certainty Newton attributes to experimental demonstrations and mathematical certainty. ${ }^{12}$

\footnotetext{
${ }^{11}$ Although Hume's account of the inductive method appears to align with Francis Bacon's account of induction, it seems as if Bacon's thinking about induction is much more embedded in matter theory than most commentators realize. Bacon argues that observations and experiments are designed to find the connection between forms and natures. Bacon's notion of nature mostly correlates with a simple sensible quality. However, the notion of form seems to relate to those active principles in corpuscles that give rise to changes in corpuscular forms. Thus, forms are not necessarily sensible qualities; they may exist below the realm of observable qualities. Moreover, while Bacon argues that observations and experiments ought to reveal that certain forms are necessary and sufficient for certain natures, he does not imagine this connection to be that of a cause-effect relation, but rather thinks of it as an identity relation between an unobservable corpuscular arrangement and observable qualities. An example would be the correlation between corpuscular motions and heat. Thus it is not clear that Hume's argument undermines even Baconian induction. I elaborate on this reading of Bacon in Belkind (Belkind).

${ }^{12}$ Based on their interpretation of Rule IV for the Study of Natural Philosophy, Ducheyne (2012, 82 n. 151, n. 152) and Walsh (2017) think that Newton takes his conclusions in the Principia to carry certainty, undermining those commentators who take Newton as thinking of his scientific theories as probable at best. According to Ducheyne and Walsh, perhaps Newton takes mathematics to be so central to his analysis of phenomena as to render his arguments non-ampliative or purely demonstrative. This claim takes its cue from the proper translation of the Latin text of Rule IV. The text is usually translated as saying that the results of inductive inferences should be taken as "either exactly or very nearly true". But the proper translation, according to Ducheyne, should be "either exactly or as most closely as possible true". This opens the possibility that Newton takes his theory to be certain and, if any corrections are required because of future observations, that the theory be made to cover a smaller scope or made less general rather than be corrected. Contrary to Ducheyne and
} 
Newton's inferences in the Principia are more complex, as he does not explain in much detail how one might derive his laws of motion from experiments and observations. Moreover, it is clear that some extrapolation and idealization takes place in articulating the laws. Moreover, in the Principia, Newton deduces from the Phenomena the centripetal nature of the force of gravity and the fact that it is inversely proportional to the distance squared. But the inference seems to be a deductive, mathematical inference, rather than a generalization from particular experiences.

Indeed we might describe the main inferences in Book III of the Principia as following a Demonstrative Inductive form of reasoning. ${ }^{13}$ According to this reasoning, one may use a phenomenal law and some background causal law or law of nature to deduce, using valid deductive arguments, other scientific propositions. From Kepler's Area Law ${ }^{14}$ and the laws of motion and with the help of Euclidean theorems, Newton deduces the centripetal nature of the force of gravity. Furthermore, from Kepler's Harmonic Rule ${ }^{15}$, the centripetal nature of the force, and from the laws of motion, Newton derives the inverse-square nature of the force. These arguments are deductive arguments that rely on phenomenal laws and laws of nature as their premises. Despite their deductive nature one may think of these arguments as inductive since they enable Newton to derive the nature of the gravitational force from the observed motions of the planets and moons in the solar system. Moreover, it seems as if Newton's argument follows a certain Baconian ascent: the initial step to the centripetal nature of the force is a low-level generalization from the phenomena. The next inference to the inverse-square nature of the force is a further ascent and an introduction of a higher level generalization, or "axiom". Thus it may be that Newton is following a Baconian project whereby induction is the gradual process by which higher and higher levels of generalizations are introduced step-wise. ${ }^{16}$ It may be that by induction Newton is referring to non-ampliative arguments that rely on mathematical reasoning that use phenomenal laws and laws of nature as their premises to introduce higher level generalizations.

Even though Hume's skeptical argument does not directly undermine arguments in the form of Demonstrative Induction, it does undermine them in indirect ways. First, in order to utilize phenomenal laws as premises in Demonstrative Inductive arguments, one may question the whether those laws can project into the future. If, for example, we consider Kepler's Area Law, we see that the law

\footnotetext{
Walsh, I think that, even with the translation corrected, the correct interpretation is that his results can be approximations to the true descriptions or are only probably true.

${ }^{13}$ For an explanation of this form of reasoning see Dorling (1973), Harper (2012), Norton (2003), and Belkind (2012).

${ }^{14}$ The Area Law states that the radius to the satellite from a central body covers equal areas in equal periods of time.

${ }^{15}$ According to Kepler's Harmonic Rule the period of completing satellite's orbit is proportional to the mean radius to the power of $3 / 2$, or $t^{2} \propto R^{3}$.

${ }^{16}$ See Ducheyne (2005) for this reading of Newton's method.
} 
articulates a correlation between two distinct aspects of a satellite's motion - the area traversed by the radius to the satellite and the time that passes. The phenomenal law projects into the future the claim that the satellites will continue to behave in the same manner, and so one could raise Humean doubts about the justification of this claim. Second, the argument in the form of Demonstrative Induction relies on causal laws or laws of nature to derive the conclusion. And, once again, one can raise Humean doubts about whether it is legitimate to extrapolate those laws from observations. The situation is exacerbated with regards to laws of nature because Newton uses the counterfactual validity of these laws in the derivation. Such validity is directly related to the type of necessity associated with the law. Since arguments in the form of Demonstrative Induction are only as good as the premises of the argument, it is legitimate to think at first glance that Hume's skeptical argument undermines Newton's reasoning, both in the Opticks and in the Principia. Nevertheless, there is a certain inductive strategy Newton uses in establishing the validity of laws of nature. It is this Newtonian inductive scheme that seems to evade Humean skepticism. In a nutshell, while Hume conceives of induction as substantiating causal claims between two sensible qualities, Newton's use of of induction is very much embedded in a corpuscular framework. Newton intends to use induction to reveal the primary qualities of corpuscles. The laws of nature are then taken to describe the types of actions that can be attributed to the most basic material corpuscles.

When Newton turns to his investigation of gravity, he expects gravity to be a sensible quality reducible to the mechanical qualities of the ether. However, in the years before writing the Principia, Newton arrives at the conclusion that gravitational effects cannot be reduced to the action of a mechanical ether. ${ }^{17}$ As he says in the General Scholium in the third book of the Principia:

Thus far I have explained the phenomena of the heavens and of our sea by the force of gravity, but I have not yet assigned a cause to gravity. Indeed, this force arises from some cause that penetrates as far as the centers of the sun and planets without any diminution of its power to act, and that acts not in proportion to the quantity of the surfaces of the particles on which it acts (as mechanical causes are wont to do) but in proportion to the quantity of solid matter, and whose action is extended everywhere to immense distances, always decreasing as the squares of the distances. (Newton, 1999, 943)

\footnotetext{
${ }^{17}$ For an account of Newton's argument for the Universal Law of Gravity, see Harper (1990, 2002, 2012), Smith (2002), Janiak (2008, 2015), and Belkind (2012).
} 
Were gravity to be reducible to the mechanical actions of an ether or a mechanical medium, we would expect the gravitational effect to depend on the area and the shape of the surfaces of bodies. But gravity does not behave in this way. The force of gravity penetrates the most inner recesses of bodies without weakening its power. Moreover, the law describing gravity is a universal law of nature, given that "gravity exists in all bodies universally and is proportional to the quantity of matter in each" (Newton, 1999, Proposition 7, 810). But how does Newton arrive at the conclusion that gravity exists in all bodies universally?

Rule IV and the explicit insistance on induction was only added in the third edition in 1726, while in the second edition (1713), induction is only mentioned in the General Scholium (Newton, 1999, 943). I will not go into differences between the various editions of the Principia, but will examine the relevant methodological rule, Rule III:

The qualities of bodies that cannot be intended and remitted [i.e., qualities that cannot be increased and diminished] and that belong to all bodies on which experiments can be made should be taken as qualities of all bodies universally. (Newton, 1999, 795)

Rule III articulates Newton's methodological rule, with the help of which one may uncover the universal qualities of matter. The rule is a summation of two criteria. A quality $Q$ is deemed universal if and only if

1. $Q$ is observed in all bodies on which experiments can be made.

2. $Q$ is invariant, i.e., it cannot be intended or remitted.

Rule III is appealed to in Book 3, Proposition 6, Corollary 2, where Newton says that all weights that were measured (that is, the weights of bodies on the surface of Earth, the weights of the planets relative to the sun, the weights of the moons of Jupiter relative to Jupiter, etc) are proportional to the quantity of matter (i.e., to mass). He claims that "This is a quality of all bodies on which experiments can be performed and therefore by rule 3 is to be affirmed of all bodies universally" (Newton, 1999, 809).

On its initial reading, Rule III reads like an inductive generalization. ${ }^{18}$ The gravitational quality was measured in all bodies on which experiments and observations were performed. Then, Newton concludes that the quality is affirmed of all bodies universally. The inference seems like an inductive

\footnotetext{
${ }^{18}$ The inductive reading of Rule III can be found in Achinstein (2010), Biener and Smeenk (2012), De Pierris (2012), and Ducheyne $(2012,117)$.
} 
argument, although one performed on a massive scale. We are looking at all bodies that were observed, and concluding regarding all bodies whatsover that they have the quality. So the inference is something like the following: "All observed bodies have quality $Q$ ", therefore, "All bodies have quality $Q$ ".

Now it might be that we have some pause regarding the notion of "body" since Newton did not provide an a priori definition of this notion. He did say at the beginning of the Principia that he associates the concept of Quantity of Matter or Mass with the notion of body. ${ }^{19}$ However, the explication of Rule III argues that impenetrability is shown to be a universal quality given that it obeys Rule III. Thus, even the universality of impenetrability and its connection with "being a body" is supposedly derived from Rule III. Furthermore, it is by virtue of impenetrability that a body has mass or Quantity of Matter. The upshot is that a body is not defined a priori as that which has mass; rather, Newton argues that empirical evidence suggests that the two are associated. We can think of Rule III as providing the criteria for determining which qualities are primary, which is indirectly the criteria for discovering the necessary qualities for being a material body. The novelty in Newton's procedure is that the more qualities that can be universalized are discovered, the more determinate we can make the notion of body. ${ }^{20}$

The reading suggested here of the notion of body does not conflict with the reading articulated by Brading (2012), according to which Newton takes the notion of body to be completed by articulating the laws of nature that apply to bodies. As we shall see shortly, the function of Rule III is to provide criteria for identifying the primary qualities of bodies. But at the same time that a quality is universalized, the law that governs a quality's action is recognized as a universal law of nature. Thus, we may see Rule III as doing two things at the same time: universalizing qualities to all bodies and

\footnotetext{
${ }^{19}$ In the explication of the definition of Quantity of Matter, Newton says, "For the present, I am not taking into account any medium, if there should be any, freely pervading the interstices between the parts of bodies. Furthermore, I mean this quantity whenever I use the term "body" or "mass" in the following pages. It can always be known from a body's weight, for - by making very accurate experiments with pendulums - I have found it to be proportioned to the weight, as will be shown below" (Newton, 1999, 404).

${ }^{20}$ It is plausible to assume that, initially, by "body" Newton means whatever it is that can generate sensory impressions on the human mind. The notion of body is that which can be manipulated in experiments or influenced so as to generate sense-impressions on the human mind, whether by direct or indirect means. This notion is confirmed by a little creation story that Newton articulates in the De Grav. In this story, Newton imagines God creates bodies by rendering certain regions of space impenetrable. He also imagines that God allows those impenetrable regions to move about, colliding and interacting according to the laws of motion dictated by God. Newton argues in the De Grav that we can take these impenetrable regions to be bodies, as long as they meet certain conditions: "(1) that they be mobile...(2) that two of this kind cannot coincide anywhere, that is, that they may be impenetrable... and (3) that they can excite various perceptions of the senses and the imagination in created minds, and conversely be moved by them" (Newton, 2004, 28-29). Given that Newton takes both mobility and impenetrability to be shown as universal by Rule III, the only a priori notion of body that is left takes a body to be that which can generate perceptions in created minds.
} 
determining which regularities can be treated as laws of nature. ${ }^{21}$

Many commentators have interpreted Rule III as an inductive inference performed on a grand scale, generalizing to all bodies. Such qualities are difficult to find, and perhaps the main difference between the type of induction that Newton considers in Rule III and the inductive arguments that Hume discusses is that Newton is concerned with a very short list of qualities, ones that 17th-century philosophers like Boyle and Locke referred to as the primary qualities of bodies. Newton lists extension, impenetrability, hardness, force of inertia, and mobility as the universal qualities. This list is more or less accepted by Newton's contemporaries as primary qualities.

However, if one reduces Rule III to universal induction, then it is not clear what role one might attribute to the invariance criterion. Thus, the argument that universalizes the quality looks as follows: All observed bodies have quality $Q$.

$Q$ is invariant.

Therefore, all bodies have quality $Q$.

In a recent paper (Belkind, 2017) I have traced the textual origins of the invariance criterion and demonstrated the relation between this criterion and Newton's corpuscularianism. The type of invariance that Newton insists on is invariance under changes of "texture" - that is, it is invariance under changes of the configuration of the body's parts. To understand this idea, we need to understand why Newton thinks that proportionality to mass has important methodological implications.

The most important evidence suggesting that gravity cannot be reduced to the mechanical properties of the ether is the fact that gravity is proportional to the mass of the gravitating body. This property of gravity is deduced from the fact that gravitational acceleration is the same for all bodies. For example, if we take several pendulums, each with a made a different material, the pendulum's period will always be the same for a particular pendulum length, no matter what the weight of the pendulum is or what it is made of. One can infer from this that gravitational acceleration on the surface of Earth is the same for all bodies. Moreover, it follows that the gravitational force is always proportional to the body's mass.

\footnotetext{
${ }^{21} \mathrm{~A}$ discussion of criteria and principles of individuation is beyond the scope the paper, which Brading takes to be relevant to the problem that is solved by taking laws to be constitutive of the notion of body. I may add that in Newton's thinking, laws of nature are constitutive of the notion of action. The individuation of action requires both laws of nature and the notions of absolute space and time. He then takes bodies to be defined by the types of actions that they generate. So laws of nature are constitutive of bodies, but only indirectly, via their constitution of actions. Moreover, the notions of impenetrability and mobility, and centripetal forces, are epistemically crucial in individuating bodies and the qualities that can be attributed to them. But we should also recognize that, while all laws of nature are constitutive of the notion of bodies, not all laws are essential in articulating criteria or principles of individuation.
} 
But if gravity is proportional to the body's mass, we can imagine that it arises from forces operating on the body's ultimate parts, and not from any particular configuration of the parts. If a property is proportional to mass and to no other bodily property, it always divides exactly as the body divides. So, for example, if the force of gravity operating on a body weighing $1 \mathrm{~kg}$ is of a certain magnitude, dividing the body into two parts, each weighing $\frac{1}{2} \mathrm{~kg}$, the force of gravity will be divided in half as well. And if the parts are divided further, the force of gravity will be divided accordingly in proportion to the masses of the parts. If we continue and divide the body further, until we reach the smallest particles from which the body is made, the force of gravity will be divided further and further, exactly as matter is divided. All of this indicates that proportionality to mass implies for Newton the invariance of the quality under variations of configuration or texture. Because the quality arises from the qualities of the ultimate parts, the contingent configuration of the parts makes no difference to the quality in the composite body.

The purpose of the invariance criterion is therefore to show the reducible nature of the gravitational force. According to Newton, we may conclude from the premises of the argument the following conclusion:

All observed bodies have quality $Q$.

$Q$ is invariant under changes of configuration.

Therefore, all ultimate parts of observed bodies have quality $Q$.

We may call this sort of inference transductive, following McGuire (1967, 1968, 1970). The transductive inference sanctions the inference from qualities of composite bodies to qualities of their ultimate parts. McGuire argues that transduction is even riskier than inductive inferences because it is an inference from observable qualities to qualities of bodies that are unobservable in principle. Newton presupposes that certain physical magnitudes behave like extensive magnitudes. Namely, these are magnitudes where the quantity correlates with the "number" of parts in a body. For example, length and volume are extensive magnitudes because the size of a body indicates the magnitude of the body. If we divide the body of a certain volume into $n$ parts, the size of the composite body would be the sum of the sizes of each of the parts. Finally, Quantity of Matter behaves like an extensive magnitude because the Quantity of Matter of a composite body is the sum of the Quantities of Matter of each of the parts. 
We see that Newton's inference is composed of two distinct inferences. ${ }^{22}$ The first inference is a transductive inference, while the second is that of a universal induction. So we may think of the combined inference as follows:

\section{Transduction}

All observed bodies have quality $Q$.

$Q$ is invariant under changes of configuration.

Therefore, all ultimate parts of observed bodies have quality $Q$.

The second inference is the following:

\section{Universal Induction}

All ultimate parts of observed bodies have quality $Q$.

Therefore, all ultimate parts of bodies have quality $Q$.

The last inference can be considered universal induction. It underwrites the notion of a primary or a universal quality. Because we have evidence that a certain quality is present in all the corpuscles from which observed matter is comprised, Newton concludes that the quality can be deemed universal. Furthermore, it is in virtue of the universality of the quality that the laws describing the action of this quality are deemed laws of nature. The purpose of Rule III is not to reveal the ultimate causes of these primary qualities, nor can it argue that all these qualities are inherent in or essential to bodies.

What can be deduced from Rule III is the quality's universal nature. ${ }^{23}$

\footnotetext{
${ }^{22}$ Achinstein (2010) argues, for example, that there are non-formal aspects of Newton's induction that must be taken into account to understand the nature of Newtonian induction. But he does not explain the relation between the non-formal aspects and the transductive inference.

${ }^{23}$ In a recent paper, Biener and Schliesser (2017) argue that there is a tension between the universality claimed by Rule III for laws of nature and a certain passage in Query 31 of the English edition of the Opticks. In the passage, Newton claims that God could have varied the sizes, shapes, densities, and forces of particles, which would as a result vary the laws of nature, and created different kinds of worlds governed by distinct laws. Biener and Schliesser claim that this suggests that laws of nature are contingent for Newton and dependent solely on God's will. It seems as if this suggestion undermines the universality insisted on in Rule III. Biener and Schliesser resolve the tension by differentiating between the context of constructing a natural philosophy versus a metaphysical context. For the purpose of grounding a science of our world, Rule III implies that certain qualities and laws are universal. The metaphysical context is one where the modality of laws of nature is explored and their contingent nature articulated. This reading seems plausible, except that it is not clear what Newton means by different worlds that exist in different regions of space - what then are the criteria by which a world is isolated or insulated from other worlds? Is there a principled way in which a particle of one world is screened off from entering another world? The law of inertia implies, at least, that particles in our world, if they are able to get detached from the attractive forces in our world, will eventually be able to penetrate any world in any direction in space. It seems to me that the suggestion in Query 31 is simply incoherent. There is no way to correlate between "worlds" and laws of nature that inhabit these worlds, unless these worlds are somehow metaphysically isolated. However, Newton does not provide any criteria for isolating or individuating worlds. It is odd that Newton would genuinely think that the universality of laws of
} 


\section{Newtonian Induction and Hume's Problem of Induc-}

\section{tion}

We shall now consider how Newtonian induction fairs under Hume's argument against the rationality of induction. An initial examination should provide a negative judgement: Hume has shown conclusively that induction is not a rational inference, and so it more forcefully applies in the case of universal induction. The scope of universal induction is wider than any inductive inference considered by Hume. The more risk the inductive argument takes, the more skeptical we should be of the conclusion. Newton's inductive inference is of a staggering scope: He claims that every single particle of matter in the universe, including particles of galaxies that exist millions of lightyears away, will have the gravitational quality. Moreover, while writing the Principia, Newton had knowledge of but a minuscule portion of the universe. He had observations of a few planets rotating around a very remote and insignificant sun, some measurements of moons rotating around Jupiter, Saturn, and Earth, and of bodies accelerating on the surface of Earth. Yet his conclusion supposedly embraces the vast expanses of the universe, to which Newton never had any access.

The immense inductive risk in Newton's argument is further exacerbated by the NRSA. Hume has shown that the inductive inference cannot be justified. If one pursues the inductive generalization, then one cannot appeal to any further arguments to show that the inductive risk is justified. So Newton's argument seems both risky and lacking any further justification.

However, the SPA seems undermined by Newton's argument. If Newton uncovered evidence that certain powers reside in the ultimate parts of matter, one cannot appeal to the SPA to demonstrate the potential failures of the inductive inference. The powers attached to the ultimate parts of matter are the secret powers that one can associate with particles. If a body can be divided, the quality is expected to divide along the resultant bodies exactly as the extensive measures of the quality are divided. Thus, it is not possible to separate the quality from the body or from its parts. Furthermore, it is not possible to diminish the quality by reconfiguring its parts. Thus, the SPA is made irrelevant by Newton's transductive argument. In virtue of the inseparability of the power from bodies (given its divisible nature), we expect the same powers to be present "all the way down", to the least bit of matter. The worry that there might be different powers that produce the same sensible qualities becomes obviated. The power in the composite body is merely the aggregate of powers present in the

nature could somehow be limited to particular regions of the universe. 
ultimate parts.

The second inference Newton applies is the universal inference from ultimate parts of examined bodies to unexamined ultimate parts. When considering the inference to the unexamined ultimate parts, one expects the power to be either present or not present in the unexamined particle. If the power is not present, there is no alternative secret power to appeal to that would explain why it is not present (it being an ultimate part). If it is indeed not present, then it is merely the capriciousness of nature that would allow for the quality to present in some ultimate parts of observable matter, but not in others. We can take the universal induction to be based on the notion that nature is not entirely capricious. In other words, the correlation that is supported by universal induction is not between a cause and its effect or between two sensible qualities. Rather, the correlation is between "being a body" and "having quality $Q$ ".

One might think that perhaps in the SPA Hume is targeting some Principle of Uniformity of Nature implicit in Newton's reasoning. ${ }^{24}$ Newton's Rule I for the Study of Natural Philosophy asserts that "we are to admit no more causes of natural things than such that are both true and sufficient to explain their appearances." It seems as if Newton is committing himself to the view that Hume targets since Hume's claim in the SPA is that we cannot take bodies to have the same powers whenever we see the same sensible qualities produced by these bodies. However, Newton's application of Rule I is not the same as the principle being attacked by Hume, according to which like causes are assumed for like effects. The way Newton uses Rule I suggests that he is not linking unknown causes with known effects. Newton uses Rule I to argue that different systems governed by the same force-laws are generated by the same forces. The forces in four distinct systems (the sun and its planets, Jupiter and its moons, Saturn and its moons, and the Earth and its moon) are governed by a centripetal, inverse square law. The effects we see are all explained by the same law governing the action of central bodies. Rule I allows Newton to conclude they are different manifestations of the same force. We might think of this as taking the law that governs the action of the force to define the nature of the force (so like effects (laws) are produced by like causes (forces)). The idea of cause-effect that Hume has in mind is entirely different. For Hume, causes are distinct from effects, and the effect is not a law but a sensible quality. In any case, the process of the universalization of qualities in itself does not rely on the principle that like effects presuppose like causes. Rather, it relies on criteria for the universalization of qualities.

\footnotetext{
${ }^{24}$ I thank one of the reviewers for pointing out this possibility.
} 
Since the SPA is made redundant by Newton's transductive argument, it follows that there is an opening for Newton to accept the inductive inference without second-order justification. According to this option, one does not seek rational justification for the inductive inference, given that universal induction is the grounds for inferring the nature of material bodies from the empirical evidence. The justification for adopting universal induction would be the same as the justification for deductive rules of inference: Namely, they are accepted in virtue of their basic status as ultimate grounds for probable inferences. Moreover, given that we have no positive reason to doubt the validity of Newtonian induction provided by the SPA, it seems rational to adopt the inductive inference.

Hume's reconstruction of the inductive inference seems ignorant of the nature of Newtonian induction. ${ }^{25}$ This is not merely due to the lack of mathematical training or understanding of Newtonian science on Hume's part, although this might contribute to Hume's misunderstanding. Rather, the problem also has to do with Newton's cagey explications of Rule III. The revisions in the Principia suggest that Newton initially presupposed a corpuscular framework as a hypothetical background to the Principia. In the first edition of the Principia Newton takes for granted that matter consists of indivisible atoms, all possessing the same kinds of power. Although he attempts to show that, in contradistinction to the mechanistic paradigm of Descartes and his followers, particles also possessed gravity, a non-mechanical quality. But the revisions Newton includes in the second edition of the Principia demonstrate that he had to reconcile his corpuscularianism with his inductivist methodology. He avoided the conflict by backing away from an explicit commitment to atomism and replacing it with a methodological rule with the help of which one can uncover the primary qualities of material corpuscles. Thus in the second edition replaces hypothesis 3, which appears in the first edition, with Rule III. The purpose of Rule III is to show that instead of making an explicit commitment to corpuscularianism, one can appeal to the extensive nature of qualities to differentiate them from secondary qualities, or qualities that are not universal in all bodies. Newton's cagey methodological remarks and his transductive inferences were understood by very few individuals.

Hazony and Schliesser (2016) argue that one way in which Hume criticizes Newton's reasoning is by replacing Newton's Rule III with a rule describing the proportionality between causes and effects, which is Rule VII in Hume's Treatise (T I.III.15.9). Hazony and Schliesser take this rule to be the one

\footnotetext{
${ }^{25}$ The relationship between Hume and Newton is complex. On the one hand, Hume describes himself in the Treatise as someone who is carrying out a Newtonian style investigation of the human mind. On the other hand, he is critical of core aspects of the Newtonian project, including the nature of absolute space and time, the reality of forces, and the universality of laws. See Schliesser (2007), De Pierris (2012), Hazony and Schliesser (2016), and Slavov (2016) for an assessment of the relation between Newton and Hume.
} 
intended to replace Rule III, because it seems to employ mathematical proportions between causes and effects, and Hume refers to the gravitational force as a compositional force, suggesting that gravity is proportional to the causes that are present in the parts of bodies (Hazony and Schliesser, 2016, 690). With the same rule, Hume seems to show that one cannot universalize gravity beyond the realm of our experience. But it is clear that Newton's invariance criterion does not describe the proportionality between causes and effects; rather, it describes the proportionality that arises from the extensive nature of qualities. This proportionality does not show us that, say, the intensity of heat is proportional to the intensity of warmth that we feel: rather, the quantity of gravity is proportional to the "number of parts" in a body or to the body's mass.

While Newton's universal induction seems to escape Hume's skeptical conclusions, there is still a worry concerning the quality of extension. Newton lists Rule III as an empirical methodological rule by which extension can be shown to be a universal quality. However, the problem is that he conceived of the invariance criterion as invariance under changes of form or, in other words, invariance under changes of the corpuscular configuration of the parts. The spatial configuration of the parts is described with Euclidean laws, that is, with the laws that are justified by the universality of extension. So, one can see that the invariance criterion presupposes the universality of extension and the necessary status of laws of geometry, while Rule III supposedly uses the invariance criterion to universalize extension. This perhaps is not a problem in Hume's account since he deems that geometry a science that belongs to relations of ideas and does not depend on any empirical considerations. However, Newton does seem to hold a dual epistemic approach toward spatial properties. First, it is an a priori science used to describe potential geometric figures to be found in absolute space. Second, the extension of bodies is taken to be universalized via Rule III, that is, via an empirical rule for revealing the primary qualities of bodies.

Once we accept that extension is a universal quality, we can think of Newton's Rule III as providing an empirical criterion for extending our knowledge of primary qualities. First, by showing that impenetrability and hardness behave like an extensive quality, Rule III demonstrates these to be universal, primary qualities. Given that Quantity of Motion is proportional to mass, or to an extensive quantity, Rule III can then demonstrate the force of inertia and mobility to be universal. Finally, relying on the nature of mass and on the counterfactual validity of laws of motion, the inferences of Book III of the Principia and Rule III demonstrate the universal nature of gravity. Thus, at each step, Rule III is responsible for broadening our understanding of primary qualities, while each step presupposes the 
previous one.

\section{Conclusion}

In my reading of Hume's argument against induction, the skeptical conclusion only follows if one combines the NRSA with the SPA. Without the SPA, the NRSA opens the door for accepting the rule of induction as a fundamental rule of inference, one that grounds probable inferences without requiring second-order justification. But with the SPA, there is enough suspicion regarding the reliability of induction to reach the skeptical conclusion.

Newton's Rule III combines two criteria for the universalization of qualities. The first criterion describes the empirical basis of the inference. Only qualities that are observed in all bodies can potentially be universalized. Second, the invariance criterion requires that a quality be invariant under changes of configuration. The two criteria lead to two inferences. First, a transductive inference: if a quality is invariant under changes of the configuration of the parts, then its quantity is divisible and it is possible to conclude that the quality is present in the ultimate parts of observable matter. The next inference is a universal inductive step - from the ultimate parts of observable matter, to all atomic parts of matter. The correlation is not between a cause and an effect, but the universal relation between "being a body" and having a certain quality. Thus, the unexamined particles of matter cannot be conceived as carrying some secret powers responsible for the same quality, given that an ultimate part of matter does not have any secret powers since the powers are directly attached to it. Thus, Newtonian induction is open to the possibility of accepting the rule of induction as a fundamental rule whose validity is accepted without further grounds. Newtonian induction, unlike most forms of induction, does not lead to the same skeptical conclusion. This is in spite of the fact that Newtonian induction is more risky than most inductive inferences because it applies to all parts of material bodies.

\section{References}

Achinstein, P. (2010). The War on Induction: Whewell Takes On Newton and Mill (Norton Takes On Everyone). Philosophy of Science 7r(5), 728-739. 
Arnold, N. S. (1983). Hume's Skepticism about Inductive Inference. Journal of the History of Philosophy $21(1), 31-56$.

Beauchamp, T. L. and T. A. Mappes (1975). Is Hume Really a Sceptic About Induction? American Philosophical Quarterly 12, 119-129.

Beauchamp, T. L. and A. Rosenberg (1981). Hume and the Problem of Causation. Oxford University Press.

Belkind, O. (Unpublished). Unnatural Acts: The Transition from Natural Principles to Laws of Nature in Early Modern Science.

Belkind, O. (2012). Newton's Scientific Method and the Universal Law of Gravitation. In E. Schliesser and A. Janiak (Eds.), Interpreting Newton: Critical Essays, Chapter 6, pp. 138-168. Cambridge University Press.

Belkind (2017). On Newtonian Induction. Philosophy of Science 84(4), 677-697.

Biener, Z. and E. Schliesser (2017). The Certainty, Modality, and Grounding of Newton's Laws. The Monist: 100(3), 311-325.

Biener, Z. and C. Smeenk (2012). Cotes' Queries: Newton's Empiricism and Conceptions of Matter. In E. Schliesser and A. Janiak (Eds.), Interpreting Newton: Critical Essays. Cambridge University Press.

Brading, K. (2012). Newton's law-constitutive approach to bodies: a reponse to Descartes. In E. Janiak A. \& Schliesser (Ed.), Interpreting Newton: Critical Essays, pp. 13-32. Cambridge University Press.

Broughton, J. (1983). Hume's Scepticism About Causal Inferences. Pacific Philosophical Quarterly $64(1), 3-18$.

Carroll, L. (1895). What the Tortoise said to Achilles. Mind 4(14), 278-280.

De Pierris, G. (2012). Newton, Locke, and Hume. In E. Schliesser and A. Janiak (Eds.), Interpreting Newton: Critical Essays, pp. 257-279. Cambridge University Press.

De Pierris, G. (2015). Ideas, Evidence, and Method: Hume's Skepticism and Naturalism concerning Knowledge and Causation. Oxford University Press. 
Dorling, J. (1973). Demonstrative Induction: Its Significant Role in the History of Physics. Philosophy of Science $40(3), 360-372$.

Ducheyne, S. (2005). Bacon's idea and newton's practice of induction. Philosophica ß 76, 115-128.

Ducheyne, S. (2012). The Main Business of Natural Philosophy: Isaac Newton's Natural-Philosophical Methodology. Springer.

Garrett, D. (1997). Cognition and Commitment in Hume's Philosophy. Oxford University Press.

Goodman, N. (1983). Fact, Fiction, Forecast (4th ed.). Harvard University Press.

Hanna, R. (2006). Rationality and Logic. MIT Press.

Harper, W. (1990). Newton's Classic Deductions from Phenomena. PSA (1990) Volume 2:, 183-196.

Harper, W. (2002). Newton's argument for universal gravitation. In I. B. Cohen and G. E. Smith (Eds.), The Cambridge Companion to Newton, pp. 174-201. Cambridge University Press.

Harper, W. (2012). Isaac Newton's Scientific Method: Turning Data into Evidence about Gravity and Cosmology. Oxford University Press.

Hazony, Y. and E. Schliesser (2016). Newton and Hume. In P. Russell (Ed.), The Oxford Handbook of Hume, Chapter 34, pp. 673-707. Oxford University Press.

Hume, D. (1975). Enquiries Concerning Understanding and Concerning the Prinicples of Morals (Third ed.). Oxford University Press.

Hume, D. (1978). A Treatise of Human Nature (Second ed.). Oxford University Press.

Janiak, A. (2008). Newton as Philosopher. Cambridge University Press.

Janiak, A. (2015). Newton. Blackwell Great Minds. Wiley-Blackwell.

Lange, M. (2008). Hume and and the Problem of Induction. In D. Gabbay, P. Thagard, and J. Woods (Eds.), Handbook of the History of Logic, Volume 10, pp. 43-91. Elsevier.

McGuire, J. E. (1967). Transmutation and Immutability: Newton's Doctrine of Physical Qualities. Ambix: The Journal of the Society for the Study of Alchemy and Early Chemistry 14(2), 69-95. 
McGuire, J. E. (1968). The Origins of Newton's Doctrine of Essential Qualities. Centaurus 12, $233-260$.

McGuire, J. E. (1970). Atoms and the 'analogy of nature': Newton's third rule of philosophizing. Studies In History and Philosophy of Science Part A 1(1), 3-58.

Millican, P. (1995). Hume's Argument Concerning Induction: Structure and Interpretation. In S. Tweyman (Ed.), David Hume: Critical Assessments: Volume II, pp. 91--144. Routledge.

Millican, P. (1998). Hume on Reason and Induction: Epistemology or Cognitive Science? Hume Studies $X X I V(1), 141-160$.

Millican, P. (2012). Hume's Scepticism' About Induction. In A. Bailey and D. O'Brien (Eds.), The Continuum Companion to Hume. Continuum.

Newton, I. (1999). The Principia: Mathematical Principles of Natural Philosophy. University of California Press.

Newton, I. (2004). Philosophical Writings. Cambridge University Press.

Norton, J. (2003). A Material Theory of Induction. Philosophy of Science 70, 647-670.

Perez Otero, M. (2008). The Humean problem of induction and Carroll's Paradox. Philosophical Studies $141(3), 357-376$.

Popper, K. (2003). The Logic of Scientific Discovery. Routledge.

Schliesser, E. (2007). Two Definitions of "Cause", Newton, and The Significance of the Humean Distinction between Natural and Philosophical Relations. Journal of Scottish Philosophy 5(1), 83-101.

Schliesser, E. (2008). Hume's Newtonianism and Anti-Newtonianism. In E. N. Zalta (Ed.), Stanford Encyclopedia of Philosophy (Winter 200 ed.). Metaphysics Research Lab, Stanford University.

Schliesser, E. (2009). Hume's Attack on Newton's Philosophy. Enlightenment and Dissent 25, 167-203.

Schliesser, E. (2017). Notes on Hume's response to problem of induction. http://digressionsnimpressions.typepad.com/digressionsimpressions/2017/05/notes-on-humesresponse-to-problem-of-induction.html 
Slavov, M. (2016). Newtonian and Non-Newtonian Elements in Hume. The Journal of Scottish Philosophy 14, 275-296.

Smith, G. E. (2002). From the Phenomena of the Ellipse to an Inverse-Square Force: Why Not? In D. Malament (Ed.), Reading Natural Philosophy: Essays in the History and Philosophy of Science and Mathematics, pp. 31-70. Open Court.

Strawson, P. (1952). An Introduction to Logical Theory. Methuen.

Van Cleve, J. (1984). Reliability, Justification, and the Problem of Induction. Midwest Studies in Philosophy 9, 555-567.

Walsh, K. (2017). Newton: From Certainty to Probability? Philosophy of Science 84(December), 866-878.

Wittgenstein, L. (1958). Philosophical Investigations. Blackwell.

Wright, C. and M. Davies (2004). On Epistemic Entitlement. Proceedings of the Aristotelian Society, Supplementary Volumes 78(May), 167-245. 